\title{
On high-latitude interstellar polarization toward the local spiral (Research Note)
}

\author{
A. Berdyugin and P. Teerikorpi \\ Tuorla Observatory, Department of Physics and Astronomy, University of Turku, Väisäläntie 20, 21500 Piikkiö, Finland \\ e-mail: pekkatee@utu.fi \\ Received 19 November 2015 / Accepted 14 January 2016

\section{ABSTRACT}

\begin{abstract}
Aims. The interstellar polarization in the northern high-latitude sky is low and rather smooth in the local spiral (LS) longitude range $60^{\circ}<l<240^{\circ}$, except for an extension of aligned higher polarization within $120^{\circ}<l<180^{\circ}$ up to $b \approx 50^{\circ}$. We compare this view with results from other indicators of interstellar matter.

Methods. Some indicators of dust are sensitive to diffuse matter on large scales, others provide information on clumpy structures. We describe results that are mainly from surveys of dark and molecular clouds in the LS longitude range at high latitudes.

Results. The structured feature in the polarization map for the LS region corresponds to known dark cloud complexes. These clouds) than is suggested by regular low interstellar polarization.
\end{abstract} \\ extensions to higher latitudes (and some other dust indicators) may signal the presence of more dust in some form (like compact cold
}

Key words. dust, extinction - local insterstellar matter

\section{Introduction}

In the 1980s, the mean extinction of light at high galactic latitudes around the galactic poles (NGP and SGP) $\left(|b| \gtrsim 50^{\circ}\right)$ was an open question that was argued to be either about 0.25 mag (de Vaucouleurs \& Buta 1983) or zero (e.g., Sandage 1988). This problem was the inspiration for the start of our interstellar polarization program in the 1990s. Measurements were gradually extended to larger distances (up to about 500 pc) and lower latitudes (Berdyugin et al. 2014 (BPT) and references therein).

BPT emphasized the impressive view in the longitude range $240^{\circ}$ to $0^{\circ}$ to $60^{\circ}$. High (up to $P=2.0 \%$ ) aligned polarizations form a giant loop centered at $l=330^{\circ}$, the upper part of the pattern that Mathewson \& Ford (1970) found from polarizations of nearby stars at lower latitudes.

Here we focus on the range $60^{\circ}-240^{\circ}$. These polarizations are low, typically $<0.1-0.2 \%$ over most of the area (Fig. 1) and the IRAS map agrees by showing weak $100 \mu \mathrm{m}$ emission. The inner edge of the local spiral (LS) arm defines this longitude zone well (Fig. 5 in BPT). The LS is about one kpc wide and populated by young stars and dark clouds. We have already (Berdyugin \& Teerikorpi 2002) emphasized that, at high north latitudes towards the LS, the interstellar polarization is low.

On the smooth low- $P$ field, there is, however, a definite extension of enhanced polarization towards higher latitudes (up to $b \approx 50^{\circ}$ around $l=120^{\circ}-170^{\circ}$ (which is not yet visible in the low-latitude map of Mathewson \& Ford (1970). In this note, we link this feature to other indications of interstellar matter towards the LS at high north latitudes. The Appendix shows the correlation between polarizations and extinctions from the IRAS map in the LS and counter directions.

\section{Interstellar high-b matter at $60^{\circ}<I<240^{\circ}$}

We outline the longitudinal behavior at latitudes $25^{\circ} \lesssim b \lesssim 60^{\circ}$ of some indicators of interstellar matter and extinction, especially dark clouds and molecular clouds.

\subsection{A bit of history: Lundmark \& Melotte dark nebulae map}

Lundmark \& Melotte (Lundmark 1927) made an early map of the distribution of 1550 so-called dark nebulae from a survey of the Franklin-Adams plates. At the time, the structure of the Milky Way was still obscure, which diminished any personal bias concerning different directions in the sky. Hence, it is not only of historical interest to show the longitudinal distribution of the dark clouds, as picked from their map between the latitudes $30^{\circ}$ and $60^{\circ}$.

In the histogram of Fig. 2, we use the old galactic coordinates as in Lundmark's map for practical reasons. We indicate below the $l^{\text {old }}$ axis the rough position of the longitude interval $60^{\circ}-240^{\circ}$. It is clear that there are concentrations of cloud detections in the LS region, one around $l^{\text {old }}=110^{\circ}$, which corresponds to $l \approx 140^{\circ}$, and another around $l^{\text {old }}=170^{\circ}$, which corresponds to $l \approx 200^{\circ}$. Still another one is found, as expected, around the Galactic center direction $\left(l(\right.$ old $\left.) \approx 330^{\circ}\right)$. We also indicate in Fig. 2 the distribution of the dark clouds in the lowest $30^{\circ}-40^{\circ}$ latitude range, where clouds at latitudes higher than $40^{\circ}$ have greatly contributed to the concentration around $l^{\text {old }}=170^{\circ}\left(l \approx 200^{\circ}\right)$.

\subsection{Dark clouds: modern results}

At lower latitudes, dark clouds (Lynds 1962; Schlosser \& Görnandt 1984; Feitzinger \& Stuewe 1986; Dobashi et al. 2005) show a similar longitudinal distribution: plenty of clouds toward the Galactic center, but also extensions toward higher northern latitudes in the general LS direction.

Schlosser \& Görnandt (1984) sketch a system of dark clouds which they call The Northern Dark Cloud System in the longitude range $90^{\circ}-210^{\circ}$. Within the range $80^{\circ}-120^{\circ}$, this is mainly at northern latitudes where they reach up to $b \approx 25^{\circ}$ around $l=110^{\circ}$ in their data. Their map also shows another extension up to $b \approx 35^{\circ}$ within the longitude range $195^{\circ}-210^{\circ}$. This could correspond to the second concentration in Lundmark's 


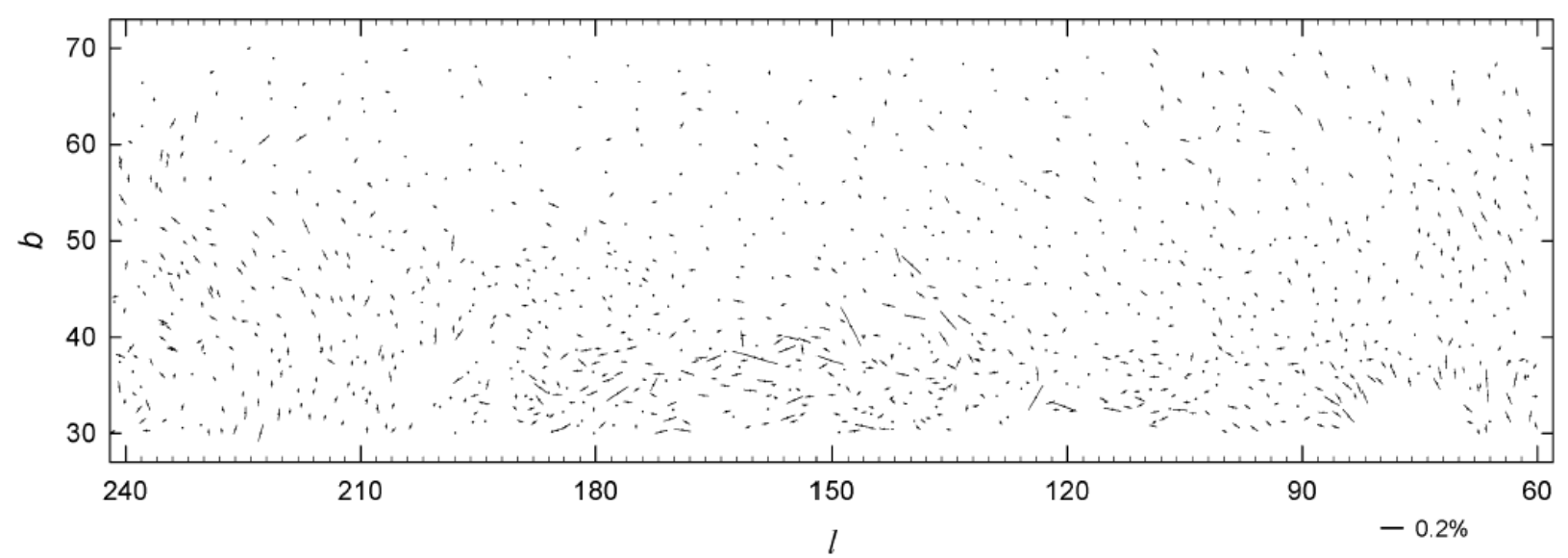

Fig. 1. Amount of polarization $P(\%)$ and its direction (the projection of polarization vector on the sky) as measured for each star is indicated by a short line proportional to the polarization percentage (or by a point, if $P=0.0$ ). From BPT.

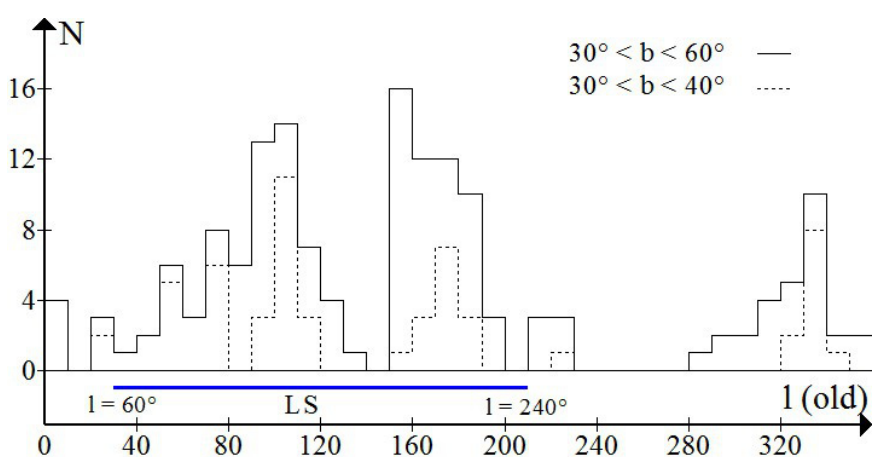

Fig. 2. Counts of high-latitude dark clouds from Lundmark \& Melotte map (Lundmark 1927). The general direction of the local spiral arm on the $x$-axis (old galactic coordinates) is shown. The Galactic center is at $l($ old $) \approx 330^{\circ}$.

map $\left(l^{\text {old }} \approx 170^{\circ}\right)$, which is not clearly visible in other maps of dust/gas indicators that we discussed.

\subsection{Molecular clouds}

Strong \& Lebrun (1982) combined Lick galaxy counts and HI observations and, assuming a fixed total gas-to-dust ratio, derived a map of the molecular hydrogen $\mathrm{H}_{2}$ column densities over the sky for $|b|>10^{\circ}, \delta>-23^{\circ}$. Interestingly, the map shows, at high northern latitudes (up to $b \approx 60^{\circ}$ ), notable amount of $\mathrm{H}_{2}$ in the longitude range $70^{\circ}<l<170^{\circ}$, and less $\mathrm{H}_{2}$ in the opposite high polarization area (the upper panel in Fig. 3. of BPT, i.e., $240^{\circ}<l<60^{\circ}$ ).

Taylor et al. (1987) studied the distribution of dark clouds where CO was detected. The so-called Galactic Belt clouds (as separated from the Gould Belt objects) showed a concentration and extension of up to $b \approx 20^{\circ}$ (their survey's latitude limit being $24^{\circ}$ ) between $l=100^{\circ}$ and $150^{\circ}$, with a peak around $140^{\circ}$. The carbon monoxide CO survey of the Milky Way by Dame et al. (2001) gives a roughly similar picture in the LS direction, with extensions of up to $b \approx 33^{\circ}$ around $l \approx 125^{\circ}$ and $\approx 150^{\circ}$.

More recently, the Planck Collaboration XXIII (2011) and Planck Collaboration XXVIII (2016) have detected a cold clump population from observations made by the Planck satellite. Their study used the three highest Planck frequency channels, 857, 545 , and $353 \mathrm{Ghz}$, where the contaminaton by the cosmic microwave background should be minimal, even at high latitudes.

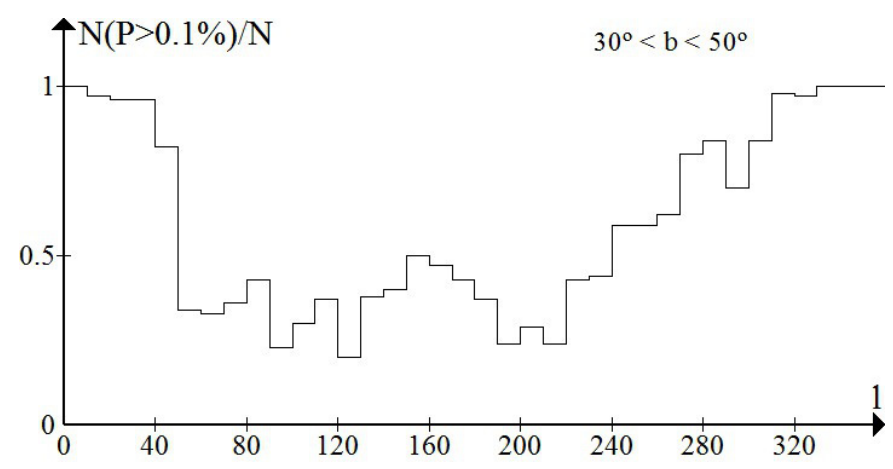

Fig. 3. Distribution of the relative number of stars with polarizations $>0.1 \%$ within the latitude range from $30^{\circ}$ to $50^{\circ}$.

The surface density distribution of the cold clump population also shows correlation with $\mathrm{CO}$ and an extension towards high latitudes $\left(b \approx 40^{\circ}\right.$ around $\left.l=120^{\circ}-150^{\circ}\right)$. The population seems to be rather heterogenous, with the mean size of the clumps $\approx 1 \mathrm{pc}$. The molecular hydrogen column density $\mathrm{NH}_{2}$ is typically $10^{20}-10^{21} \mathrm{~cm}^{-2}$.

Planck Collaboration XXVIII (2016) estimates the distances of some molecular complexes (their Table 3 ). At a distance of $380 \mathrm{pc}$, the well-known Polaris Flare $\left(l=123^{\circ}, b=24^{\circ}\right)$, which was recently well studied in polarimetry by Panopoulou et al. (2015), has a too low latitude to be visible in our map where we do not have measurements below $b \approx 32^{\circ}$ around these longitudes. With the highest latitude, the Ursa Major complex $\left(l=148^{\circ}, b=35^{\circ}\right)$ is at a distance of about $350 \mathrm{pc}$. The lower part of the Ursa Major complex is also seen in the CO map of Dame et al. 2001. The enhanced interstellar polarizations around this direction in Fig. 1 are likely due to this complex and others in the same region within the LS.

Teerikorpi (1981) studied the reddenings of quasars and their $E(B-V)$ vs. NHI relation. Above the usual relation, he found a branch of higher reddenings, especially in the LS direction. This was interpreted as being due to high latitude molecular clouds with sizes less than around $2 \mathrm{pc}$ and typical extinctions $a_{V} \gtrsim$ 0.5 mag. With such properties, these objects may relate to the clouds that were then found from their $\mathrm{CO}$ emission by Blitz et al. (1984) and to the Planck cold clumps. In fact, Cohen et al. (1983) showed that molecular clouds outline the spiral structure, including the LS. 


\subsection{Other indicators}

Also other evidence for enhanced high-latitude extinction in the LS direction exist. The reddening map from OB stars by Lucke (1978), shows the enhancement of extinction at high latitudes (up to and even above $40^{\circ}$ ) around $l \approx 120^{\circ}$. The cited quasar reddenings (Teerikorpi 1981), their correlations with the Lick galaxy counts (Haarala \& Teerikorpi 1986), and the PSA counts (Teerikorpi \& Kotilainen 1988), and the counts themselves (Teerikorpi \& Haarala 1987), also point to enhanced extinction at the high-latitude LS region.

Still another kind of dust indicator is the polarized thermal radiation from the dust itself. Recently, Planck Collaboration Int. XIX (2015) published maps of the Galactic polarized thermal emission at $353 \mathrm{GHz}$. At high north galactic latitudes, these agree with our interstellar polarization map by showing a similar asymmetry, with the polarized emission extending to high latitudes in the counter-LS direction, while showing no, or little, detected emission above $b \approx 50^{\circ}$ in the LS direction. Also, the region of detected polarization has a broad maximum around $l \approx 135^{\circ}$, where it extends up to $b \approx 45^{\circ}$. We note that the directions of the magnetic field lines in the Planck map roughly agree with those from interstellar polarization in our maps (BPT). Clearly, instellar polarization and polarized thermal dust emission are important, complementary ways of studying magnetic field directions at high latitudes.

\section{Concluding remarks}

Dark and/or molecular clouds are distributed towards high latitudes in the middle parts of the LS longitude range $60^{\circ}-240^{\circ}$. This is especially clear around $l \approx 120^{\circ}-150^{\circ}$, where the interstellar polarization is also higher than the generally low level in the LS direction and shows aligned patterns up to $b \approx 50^{\circ}$. Another concentration around $l \approx 200^{\circ}$, seen in the old Lundmark's map of dark clouds, is also visible in the dark cloud distribution derived by Schlosser \& Görnandt (1984), but not in our interstellar polarization data (see Fig. 3). It may show up weakly in Planck's cold clump map.

Possible indications for "extra" extinction at high latitudes may relate to a clumpy medium connected with the local spiral structure and which can be missed by methods suitable for detecting especially diffuse medium. On the other hand, other factors can hamper obtaining an unbiased picture at high latitudes. For example, the detections of dark clouds are increasingly incomplete owing to the decreasing stellar surface number density.
The asymmetry in optical polarizations is mainly a local phenomenon, in particular the big northern "magnetic loop" that was explained as an expanding gas/dust shell from a supernova at tens of parsecs from the Sun (Weaver 1979; see Fig. 9 in BPT for a more recent model).

In the counter (LS) direction, we look through a part of the less prominent Local Bubble and the polarizations that arise from the not so abundant diffuse dust within the LS are low, which can also be partly due to a weaker or less organized magnetic field within the spiral arm. We know that the regular component of the galactic magnetic field is stronger between the arms (Han \& Qiao 1994; Indrani \& Deshbande 1999).

Acknowledgements. We thank the anonymous referee for useful remarks and suggestions. This work was partly supported by the ERCA grant HotMol (ERC-2011-AdG291659).

\section{References}

Berdyugin, A., \& Teerikorpi, P. 2002, A\&A, 384, 1050

Berdyugin, A., Piirola, V., \& Teerikorpi, P. 2014, A\&A, 561, A24 (BPT)

Blitz, L., Magnani, L., \& Mundy, L. 1984, ApJ, 282, L9

Cohen, R. S., Cong, H., Dame, T. M., \& Thaddeus, P. 1983, ApJ, 239, L53

Dame, T. M., Hartmann, D., \& Thaddeus, P. 2001, ApJ, 547, 792

Dobashi, K., Uehara, H., Kandori, R., et al. 2005, PASJ, 57, 1

Feitzinger, J. V., \& Stuewe, J. A. 1986, Vistas Astron., 29, 291

Haarala, S., \& Teerikorpi, P. 1986, Ap\&SS, 122, 163

Han, J. L., \& Qiao, G. J. 1994, A\&A, 288, 759

Hiltner, W. A. 1956, ApJS, 2, 389

Indrani, C., \& Deshpande, A. A. 1999, New Astron., 4, 33

Lucke, P. B. 1978, A\&A, 64, 367

Lundmark, K. 1927, Medd. Astron. Obs. Upsala, 30

Lynds, B. T. 1962, ApJS, 7, 1

Mathewson, D. S., \& Ford, V. L. 1970, Mem. Roy. Astron. Soc., 74, 139

Panopoulou, G., Tassis, K., Blinov, D., et al. 2015, MNRAS, 452, 715

Planck Collaboration XXIII. 2011, A\&A, 536, A23

Planck Collaboration Int. XIX. 2015, A\&A, 576, A104

Planck Collaboration XXVIII. 2016, A\&A, in press, DOI: $10.1051 / 0004-6361 / 201525819$

Sandage, A. 1988, ARA\&A, 26, 561

Schlosser, W., \& Görnandt, V. 1984, A\&A, 137, 287

Schlegel, D. J., Finkbeiner, D. P., \& Davis, M. 1998, A\&A, 500, 525

Strong, A. W., \& Lebrun, F. 1982, A\&A, 105, 159

Taylor, D. K., Dickman, R. L., \& Scoville, N. Z. 1987, ApJ, 315, 104

Teerikorpi, P. 1981, A\&A, 98, 300

Teerikorpi, P., \& Haarala, S. 1987, Ap\&SS, 137, 397

Teerikorpi, P., \& Kotilainen, J. 1988, Ap\&SS, 155, 19

De Vaucouleurs, de G., \& Buta, R. 1983, AJ, 88, 939

Weaver, H. 1979, in The large-scale characteristics of the Galaxy, IAU Symp., 84,295 


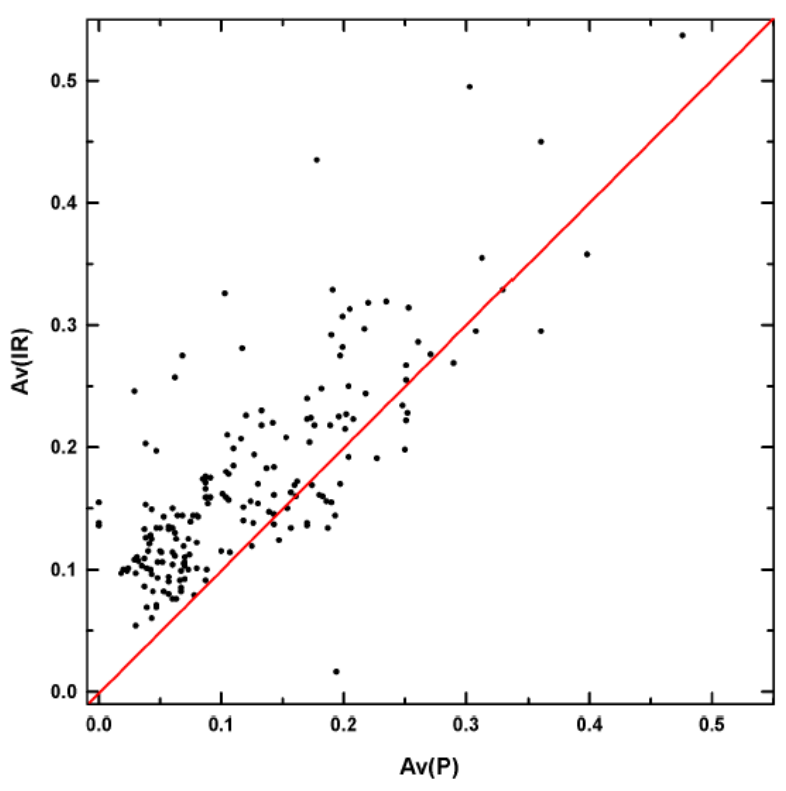

Fig. A.1. Extinction from the IR data vs. estimate of the lower limit of extinction from the interstellar polarization for the range $240^{\circ}<l<$ $60^{\circ}$. The line of equal values is shown. We note that our sample stars obey the observational criterion $P / \sigma_{P}>2$, so that the error $\sigma_{P}$ is always less than $P / 2$, being typically $\approx P / 4$.

\section{Appendix A: Polarization versus far-IR emission}

Optical polarization and $100 \mu \mathrm{m}$ emission around the NGP correlate (Berdyugin \& Teerikorpi 2002). Here we compare polarization (from BPT) at high north latitudes and extinction $A_{V}(I R)$ (Schlegel et al. 1998) from $100 \mu \mathrm{m}$ data (IRAS \& COBE/DIRBE; irsa.ipac.caltech.edu). The current maps, based on IR emission, give $A_{V} \approx 0.05$ for the areas around the Galactic poles.

We picked 190 stars inside $295^{\circ} \times 5^{\circ}$ squares in the $l$-range $240^{\circ}-60^{\circ}$ where the polarization is regular and coaligned, and 77 stars in 13 areas within the LS range $60^{\circ}-240^{\circ}$. Figures A.1 and A.2 show the corresponding $A_{V}(I R)$ vs. the lower limit $A_{V}(P)$ diagrams where $A_{V}$ is estimated as $=P_{V} / 3$, corresponding to the ideal polarization case in Hiltner's inequality $P_{V} \leq 3 A_{V}$ (Hiltner 1956).

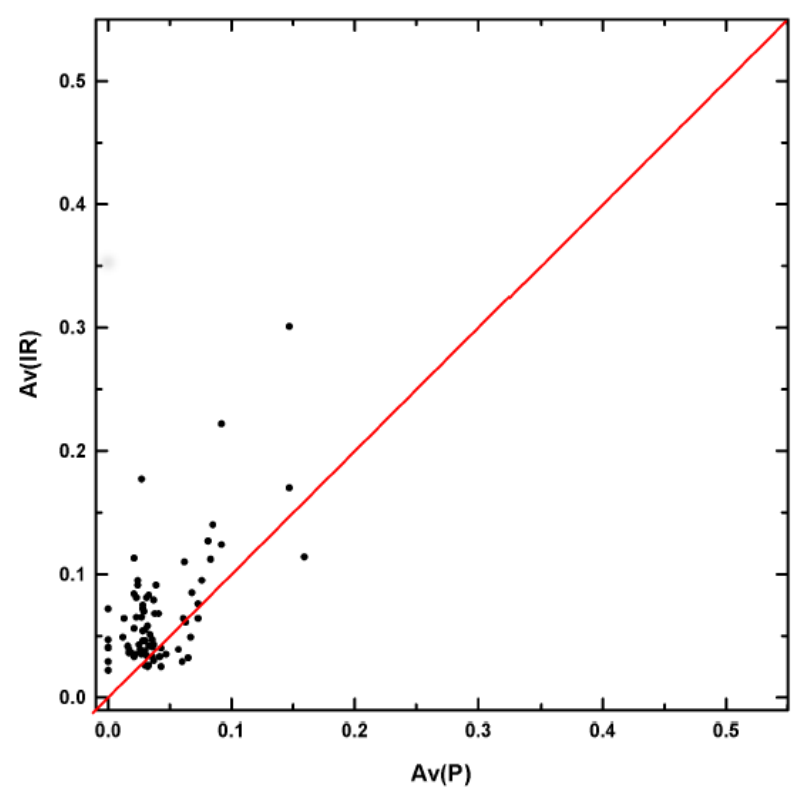

Fig. A.2. As in Fig. A.1, but for the LS range $60^{\circ}<l<240^{\circ}$.

Polarizations and IRAS data are correlated roughly as expected from Hiltner's inequality. This is the case for both the general directions, although the average polarization and IR emission is lower for the LS longitude range. A great majority of polarizations are lower than $3 A_{V}(I R)$ which approaches an upper envelope to $P_{V}$. An additional factor may be that the polarimetry probes dust only up to a certain distance $(\approx 400 \mathrm{pc})$. Also, our observations were in white light and may slightly (by 15-20\%) under-estimate the polarization in the $V$-band where interstellar polarization typically peaks.

Indeed, the slope of $A_{V}(\mathrm{IR})$ vs. $A_{V}(P)$ may be a little steeper than in the opposite zone in the LS region, with large polarization, meaning a higher ratio $A_{V}(\mathrm{IR}) / A_{V}(P)$ in the sky area with small and mostly irregular polarization ${ }^{1}$. However, this effect is small and uncertain.

\footnotetext{
1 We note that in BPT the giant loop itself displays an asymmetry: the polarizations in the west branch $\left(0^{\circ}<l<45^{\circ}\right)$ are much higher than in the east $\left(255^{\circ}<l<300^{\circ}\right)$. We conclude from the IR emission and radio polarization maps that the smaller polarization in the east is not due to little dust, but a weak (irregular) magnetic field. Here also weak polarizations do not imply little dust, not even of a diffuse kind.
} 